\title{
Óxido nítrico e exercício físico: potencialidades para a pesquisa em saúde - Artigo de Revisão
}

\author{
Nitric oxide and physical exercise: potentialities for research \\ in health - Review article
}

\author{
Nestor Pérsio Alvim Agrícola ${ }^{1}$ \\ Lídia Andreu Guillo² \\ Regisnei Aparecido de Oliveira Silva ${ }^{3}$
}

\section{RESUMO}

Este texto é uma revisão sobre os estudos que relacionam o óxido nítrico e o exercício físico. As bases de dados consultadas foram Pubmed, Scielo e Medline. O objetivo é expor os resultados de estudos envolvendo o óxido nítrico (NO), o exercício físico e sua relação com a saúde humana. O NO é um radical livre que está envolvido em uma gama de reações no organismo humano e tem a capacidade tanto de ser benéfico, quanto potencialmente tóxico. Atua de forma direta na vasodilatação e possui a propriedade de inibição da agreg-ação plaquetária. Os mecanismos protetores induzidos pelo exercício estão ligados a um aumento da síntese endotelial de NO. A associação entre o NO sanguíneo e o salivar é o novo desafio dos pesquisadores dedicados ao estudo desde composto e sua ação no organismo.

\section{PALAVRAS-CHAVE}

Óxido nítrico; Exercício físico; Saúde humana.

\footnotetext{
${ }^{1}$ Universidade Federal de Goiás / Regional Jataí, Departamento de Educação Física.

2Universidade Federal de Goiás / Instituto de Ciências Biológicas.

${ }^{3}$ Universidade Federal de Goias / Regional Jatai, Departamento de ciências Biológicas.
} 


\section{ABSTRACT}

This text is a review of the studies that relate nitric oxide and physical exercise. The databases consulted were Pubmed, Scielo and Medline. The objective is to expose the results of studies involving nitric oxide (NO), physical exercise and its relation with human health. NO is a free radical that is involved in a range of reactions in the human body and has the capacity to be both beneficial and potentially toxic. It acts directly on vasodilation and has the property of inhibition of platelet aggregation.

Exercise-induced protective mechanisms are linked to increased endothelial NO synthesis. The association between blood NO and salivary is the new challenge of researchers dedicated to the study since compound and its action in the body.

\section{KEYWORDS}

Nitric oxide; Physical exercise; Human health. 


\section{INTRODUÇÃO}

O óxido nítrico é uma molécula inorgânica, de baixo período de meia vida, gasosa e que possui um elétron desemparelhado em sua última camada, tornando-a uma molécula iônica altamente reativa que se combina facilmente com o oxigênio e com alguns metais de transição. É considerado um radical livre que pode ser tanto agente oxidante quanto redutor, dependendo do meio em que se encontra, e sua reação com o oxigênio gera nitritos e nitratos (CERQUEIRA \& YOSHIDA, 2002). Em condições normais de temperatura e pressão o NO é um gás, incolor com moderada solubilidade em água, e que reage com alguns metais de transição, entre eles o ferro. Sua forte reatividade com o ferro ocasiona a ligação do $\mathrm{NO}$ com as metalo-proteínas (proteínas com metais de transição em sua composição), entre elas a hemoglobina que contem ferro em seu grupo heme (DUSSE, VIEIRA \& CARVALHO, 2003). Apesar do potencial citotóxico, que teria como prejuízo imediato as células sanguíneas, o NO, devido ao seu breve período de meia vida, assume o papel de mensageiro químico, ativando ou inibindo outras moléculas principalmente do sistema imunológico, e não é armazenado in-vivo no organismo, e sim sintetizado por demanda, atacando os sítios invasores com seu alto poder oxidativo, principalmente células tumorais e bactérias.

Até meados dos anos 1980, não era conhecida a ação biológica do NO para o organismo humano, sendo, até então, considerado apenas membro de uma família de poluentes ambientais indesejáveis (DUSSE, VIEIRA \& CARVALHO, 2003). A investigação acerca de um fator vasodilatador determinante no relaxamento do endotélio vascular levou à hipótese da existência do Endothelial - Derivated Relaxing Factor (EDRF) (FURCHGOTT \& ZAWADZKI, 1980). Mais tarde descobriu-se que o mecanismo pelo qual a EDRF produzia o relaxamento muscular do endotélio era mediado pela Guanosina monofosfato cíclica (GMPC) (RAPAPORT \& MURAD, 1983), e a partir daí concluiu-se que o NO era a molécula comum aos processos vasodilatadores, que resultava no relaxamento muscular do endotélio, melhorava o suprimento sanguíneo ao coração e aliviava sintomas como angina (DUSSE, VIEIRA \& CARVALHO, 2003).
O NO está envolvido em uma gama de reações no organismo humano e tem a capacidade tanto de ser benéfico, quanto potencialmente tóxico, conforme sua concentração. É produzido de forma endógena por uma variedade de tipos diferentes de células. O NO é o principal mediador citotóxico de células imunes no organismo, sendo o precursor de nitritos e nitratos excretados pelos macrófagos em respostas imunológicas específicas (SNYDERS \& BREDT, 1992). Embora alguns estudos iniciais demonstrassem que nitritos $\left(\mathrm{NO}_{2}^{-}\right)$e nitratos $\left(\mathrm{NO}_{3}^{-}\right)$convertidos a partir do $\mathrm{NO}$ e de uma dieta rica em nitrosaminas em humanos e ratos poderiam ser cancerígenos, mais tarde foi demonstrado que, mesmo diante de uma dieta pobre em nitrosaminas, o organismo continuou excretando altas taxas de nitratos, comprovando assim a produção endógena no óxido nítrico (SNYDERS \& BREDT, 1992). O potencial citotóxico do NO se revela oportuno na medida em que é produzido em quantidades significativas durante a resposta imunológica e provoca danos oxidativos letais às células-alvo (BARRETO \& CORREIA, 2005). Faz parte da primeira linha de defesa do organismo com seu poder microbicida. Nessa função, atua em concentrações muito maiores que a de mensageiro.

O óxido nítrico além de atuar de forma direta na vasodilatação, ainda possui a propriedade de inibição da agregação plaquetária, prevenindo a formação de trombos. Inibe também a adesão plaquetária e leucocitária, de forma geral na parede dos vasos (FLORA FILHO \& ZILBERSTEIN, 2000). Atuando de forma não muito bem conhecida ainda, exerce ação também modificando a diapedese neutrofílica e diminuindo a permeabilidade vascular do endotélio, impedindo com isso, a circulação de células inflamatórias (KUBES \& GRANGER, 1992).

Por outro lado, existem estudos que demonstram que o NO contribui para agravar algumas condições patológicas, como asma, tuberculose, artrite reumatóide, esclerose múltipla, Alzheimer, que teriam na produção de NO um complicador, principalmente na situação de produção excessiva dessa molécula. Além dessas condições citadas, em estresse oxidativo o NO se torna um poderoso liberador de espécies reativas do oxigênio. A variedade de interações e ações possíveis dessa molécula lhe rendeu o título de 
"molécula do ano" em 1992 na revista Science, quando os estudos, envolvendo uma diversidade de hipóteses, se multiplicavam na comunidade científica (KOSHLAND, 1992).

Para esta revisão foram consultadas as bases de dados Pubmed, Scielo e Medline utilizando como descritores principais óxido nítrico e exercício físico. Os trabalhos selecionados não tiveram limites de datas, mas foram escolhidos em função da proximidade com o tema deste texto.

\section{SÍNTESE E AÇÃO}

O óxido nítrico é produzido pelas enzimas óxido nítrico sintase (NOS) que são hemeproteínas divididas em três isoformas que possuem o mesmo mecanismo catalítico e utilizam os mesmos substratos, co-fatores e co-substratos. A isoforma 1, nNOS, ou óxido nítrico sintase neuronal, é constitutiva e está presente em neurônios principalmente e no órgão sexual masculino, é cálcio-calmodulina dependente e regula a transmissão sináptica no sistema nervoso central. Atua na regulação central da pressão sanguí-
No entanto, se a expressão não for parte de resposta imunológica a um patógeno invasor, pode ser extremamente prejudicial a saúde e até sintoma de doença autoimune (CERQUEIRA \& YOSHIDA, 2002).

A isoforma 3, também constitutiva, é a óxido nítrico sintase endotelial (eNOS), que produz o NO continuamente no endotélio em condições basais. $\mathrm{O}$ aumento do fluxo sanguíneo, ao produzir maior atrito (shear estress), leva a eNOS a aumentar a produção de NO. Também é cálcio-calmodulina dependente (CERQUEIRA \& YOSHIDA, 2002). Atua na regulação da pressão sanguínea e do tônus vascular da musculatura lisa, inibe a proliferação de células da musculatura lisa das artérias, e ainda exerce influencia na contratilidade da musculatura cardíaca (MURREL, et al, 1996). A inibição da eNOS pode provocar aumento da permeabilidade vascular e acentuar a agregação plaquetária e a aderência de leucócitos ao endotélio. A eNOS fica estrategicamente ancorada à membrana da célula endotelial, em estruturas denominadas cavéolas localizadas na membrana plasmática e favorece a liberação de quantidades suficientes de NO para a camada muscular do vaso sanguíneo (DIAS; NEGRÃO; KRIEGER, 2011).

As isoformas constitutivas da NO sintase são ativadas quando ocorre um aumento na concentração de cálcio em resposta à ativação de receptores da superfície celular. Quando a concentração de cálcio intracelular cai, se desfaz o complexo cálcio-calmodulina e ocorre a desativação da enzima (nNOS e eNOS). A reação de formação do óxido nítrico se dá a partir da L-arginina, como substrato, que tem como co-produto o NO e a L-citrulina ao final. A L-arginina é um aminoácido semi-essencial produzido no organismo, também utilizado no ciclo da uréia, a partir de proteínas ingeridas e degradas e da neo-síntese a partir da citrulina (FLORA FILHO \& ZILBERSTEIN, 2000). Na reação de conversão da L-arginina em NO e L-citrulina (figura 1) também é necessário a presença de $\mathrm{O}_{2}$ e nicotinamida-adenina-dinucleotídeo-fosfato-hidrgênio (NADPH) como co-substratos. duais. Se a expressão for parte de uma resposta

\section{L-arginina}

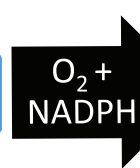

N-hidroxi-L-arginina

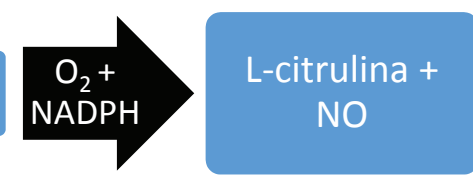

Figura 1: Reação catalisada pela NOS 
A produção de NO se dá em sua maior parte nos vasos sanguíneos. Em artérias e arteríolas a produção é maior que em veias e vênulas e a vasodilatação é maior em artérias de maior calibre (CERQUEIRA \& YOSHIDA, 2002).

Após ser produzido nas células do endotélio, o NO migra para o interior da célula muscular lisa, onde irá exercer a ação vasodilatadora. A ação do NO no pro- cesso vasodilatador envolve a interação com a enzima guanilato ciclase (GC) ativando-a. A guanilato ciclase ativada irá catalisar a molécula de guanosina trifosfato (GTP), fazendo-a perder dois grupos fosfatos, e se tornar guanosina monofostato cíclica (GMPc). $\mathrm{O}$ aumento na quantidade de GMPc irá levar a um relaxamento da célula muscular pelo aumento na concentração de cálcio intracelular (CONGER, 1994).

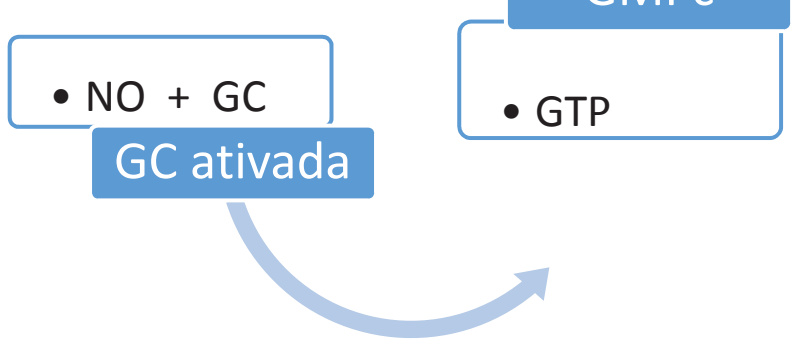

Figura 2: Ação do NO no relaxamento muscular liso

É necessário que os íons de cálcio $\left(\mathrm{Ca}^{++}\right)$estejam disponíveis para a realização do processo e a queda na sua concentração irá levar à desativação da NOS. Após a reação, o NO restante deixa a célula muscular e migra para a corrente sanguínea, onde irá penetrar nas plaquetas e hemácias. No interior das plaquetas ocorre o mesmo processo descrito na célula muscular lisa, aumento na GMPc e diminuição de íons cálcio livres, requeridos para o interior da célula, mais especificamente para o retículo endoplasmático (DUSSE, VIEIRA \& CARVALHO, 2003). Como os íons cálcio são essenciais na ativação das plaquetas, essa atividade será em parte inibida. O NO que penetra as hemácias será eliminado na forma de nitrato e nitrito, pela reação com o ferro presente na célula.

\section{DOADORES E PRECURSORES DE NO}

Com a conclusão de que o NO é uma molécula extremamente importante para diversas funções do organismo humano, a possibilidade de encontrar formas de potencializar ou otimizar a entrega deste composto ao organismo passou a ser tema de diversas pesquisas (BARRETO \& CORREIA, 2005). O que tem se verifica é que há uma grande diversidade de estruturas químicas capazes de gerar NO. Dentre os compostos mais estudados estão os nitratos orgânicos, que apresentam grande potencial liberador de NO, como a nitroglicerina (TNG) e o nitroprussiato de sódio (NPS), que possuem severas limitações ao seu uso clínico e principalmente terapêutico, que confrontam com seus benefícios (PAULO, 2011). A TNG, quando utilizada em tratamento, gera tolerância e drástica perda de seu poder doador de $\mathrm{NO}$ e vasodilatador por consequência. O NPS, depois de catalisado por enzimas específicas, além de liberar NO, libera também cianeto que é altamente tóxico ao organismo humano e ainda promove queda drástica e rápida da pressão arterial. Assim, esses compostos não têm sido utilizados com uso terapêutico, restringindo-se somente ao uso hospitalar por requererem suporte de emergência (PAULO, 2011).

Além desses, outros nitratos orgânicos devem ser citados, como o nicorandil, o dinitrato de isosorbida 
(DNIS) e o tetranitrato de pentaeritritila (TNPE), que em meios altamente alcalinos sofrem hidrólise fornecendo, a partir de atividade enzimática específica, os compostos necessários à ativação do complexo GC - GMPc. Outras classes de doadores de NO conhecidas são: N-nitrosaminas, S-nitrisotiois e N-hidroxiguanidinas e complexos nitrosilos- rutênio, que podem gerar NO a partir de, enzimas específicas, hidrólise, redução, oxidação, calor e até luz (BARRETO \& CORREIA, 2005), (PAULO, 2011).

A busca por doadores de $\mathrm{NO}$ com potenciais usos terapêuticos levou ao incremento das pesquisas envolvendo o óxido nítrico e sua diversidade de funções. Dentre as descobertas mais recentes, deve-se destacar o terpy, composto inorgânico da classe dos nitrosil-rutênio, libera NO após metabolização celular, é estável em PH fisiológico e atóxico nas condições testadas. Apresenta resposta hipotensora lenta e de longa duração, em ratos hipertensos e não leva à taquicardia reflexa, como os nitratos orgânicos (PAULO, 2011).

Outra inovação recente entre os doadores de NO estão as NO-aspirinas que consiste na hibridação de porções liberadoras de NO em drogas comercialmente disponíveis, reduzindo, com isso, a toxicidade e potencializando a atividade biológica. As NO-aspirinas não apresentam gastrotoxicidade e tem forte propriedade antitrombótica, agindo diretamente na agregação plaquetária, tem sido recomendadas para tratamento terapêutico de longa duração na prevenção de infarto e em pacientes com artrite (BARRETO \& CORREIA, 2005).

Além do uso terapêutico, as pesquisas em doadores e precursores de NO buscam meios de aumentar o desempenho atlético esportivo. A busca mais comum entre os precursores de NO, ligados ao desempenho atlético, se firma na hipótese de que o óxido nítrico está ligado à melhora da perfusão sanguínea ao nível muscular, contribuindo para maior aporte de nutrientes e de oxigênio, ao mesmo tempo em que atua como sinalizador no sistema imune e na rede neuronal (PAULO, 2011). Suplementos protéicos ricos em determinados aminoácidos são teoricamente precursores da produção de Óxido Nítrico no organismo. As proteínas ingeridas são degradadas até arginina, que podem ser diretamente absor- vidas ou transformadas em ornitina que, juntamente com a glutamina secretada como glutamato, são convertidas em citrulina. A citrulina absorvida se transforma em arginina no ciclo da ureia (BARRETO \&CORREIA, 2005). Entretanto, ainda na há confirmação dessa hipótese e os doadores de NO, relacionados ao desempenho físico, permanecem no campo experimental.

\section{NO E EXERCÍCIO}

A medição da concentração dos níveis de óxido nítrico raramente se dá pela detecção do gás propriamente dito, devido ao seu curto período de meia vida. Em geral essa medição se faz pela quantificação do produto da reação de oxidação do NO, nitratos e nitritos, que são mais estáveis, pelo composto precursor da formação do NO, a L-arginina, ou por seus subprodutos como a L-citrulina ou a guanosina monofosfato cíclica (GMPc). Também pela expressão gênica da enzima NOS. Os diversos estudos que abordam a questão da concentração de NO em exercício e seus desdobramentos se valem desses parâmetros acima mencionados, cada um deles diferindo no que tange à metodologia e ao protocolo utilizado. Esse talvez seja o fator fundamental da divergência verificada na literatura quanto aos resultados de estudos dessa natureza.

O exercício físico é caracterizado por uma saída do organismo de seu estado de homeostase, pela demanda energética imediata que ocorre na musculatura, de modo geral. A quantidade de seções semanais de exercício, o tempo de exercício e a intensidade com que é realizado parecem interferir nos efeitos hipotensores (HAMER, 2006). A diminuição da pressão arterial produzida pelo exercício diz respeito, tanto ao efeito hipotensor pós exercício, que perdura pelo tempo de recuperação, quanto pelo efeito anti-hipertensivo duradouro, que se verifica em indivíduos treinados (BRUM et al, 2000). Verificase, no entanto, que o efeito de controle da pressão arterial ocorre mais acentuadamente quando o exercício é de intensidade moderada, em torno de 50 a $65 \%$ da intensidade máxima (FORJAZ et al, 1998).

Alguns resultados parecem apontar conclusões seguras, tais como: os exercícios moderados, abaixo 
do limiar anaeróbico, realizados ate por volta de $65 \%$ da capacidade máxima de esforço, tem melhores resultados na produção de $\mathrm{NO}$ e consequentemente nos efeitos que este composto pode proporcionar. Isto porque o esforço mais elevado gera uma produção de metabólitos indesejáveis que caracterizam o chamado stresse oxidativo, com efeitos antagônicos aos apontados por concentrações ótimas de NO. As atividades físicas que envolvem grandes grupamentos musculares, como caminhadas, corridas e natação, também se destacam em relação a exercícios mais localizados. Isso devido ao aumento da frequência cardíaca, do volume sistólico e do débito cardíaco que em atividades desse tipo são mais pronunciados (BRANDÃO \& MARTINS-PINGE, 2007).

Estudos recentes tem demonstrado a relação do perfil lipídico, produção do NO pelo endotélio e exercícios físicos. O exercício é apontado como importante fator regulador das dislipidemias uma vez que o aumento do fluxo sanguíneo e a consequente maior produção de NO pelo endotélio ajudam a regular os níveis de LDL e HDL colesterol (DREXLER, 1999), (KINGWELL, 2000). A maior produção de NO em exercício físico, decorrente de aumento no shear stress foi verificada tanto em indivíduos normotensos quanto hipertensos e por isso o exercício é apontado como restaurador da função endotelial em casos de disfunção associadas a doenças cardiovasculares (ZAGO \& ZANESCO, 2006).

O óxido nítrico é um mediador da microcirculação local do miócito, atuando no fornecimento de energia do músculo esquelético através da modulação hormonal, principalmente do hormônio GH. Segundo estudo recente (DYAKOVA et al, 2015), o NO atua na ativação da biogênese mitocondrial do músculo esquelético, melhorando a respiração do miócito. Interfere diretamente no abastecimento de nutrientes e oxigênio do miócito através da regulação do fluxo sanguíneo. A exposição a longo prazo ao NO, por células musculares, desencadeia a biogênese mitocondrial envolvendo a ativação da GMPc (DYAKOVA et al, 2015). Outros estudos vem apontar a relação do exercício físico com doenças como Parkinson e Alzheimer mediadas pela produção de NO no organismo (PAILLARD, ROLLAND \& BARRETO, 2015). O exercício físico de caráter aeróbico ativa a liberação de fatores neu- tróficos e promove a angiogênese, facilitando a neurogênese e a sinaptogenese que por sua vez melhoram as funções cognitivas. Os estudos citados acima demonstraram que os mecanismos protetores induzidos pelo exercício estão ligados a um aumento da síntese endotelial de NO e ainda um aumento do fator de crescimento endotelial vascular. Os autores ainda afirmam que as doenças neurológicas estão em crescimento em nossa sociedade e uma simples ação de incentivo à atividade física poderia trazer um impacto positivo na saúde pública.

\section{NO SALIVAR E EXERCíCIO}

A saliva tem sido muito utilizada na quantificação do óxido nítrico principalmente em estudo da cavidade oral, relacionados a doenças periodontais, implantes dentários, carcinomas, etc. principalmente devido à facilidade que a técnica oferece. A saliva pode ser coletada por pessoal não especializado por não ser um processo invasivo, e a técnica de quantificação colorimétrica de nitrito usando o reagente de Griess é relativamente rápida e de custo não tão elevado, possibilitando a testagem de uma variedade de hipóteses. No campo da atividade física, a saliva tem sido utilizada na busca de se estabelecer um protocolo seguro e confiável para a testagem de respostas fisiológicas do organismo ao esforço e a definição de parâmetros de controle do exercício que sejam não invasivos (ZUARDI, 2012)

A saliva é produzida pelas glândulas parótidas, submandibulares e sublinguais, todas bilaterais. Alem dessas, a saliva também é produzida por glândulas microscópicas localizadas nas regiões labial, lingual, palatina e retromolar. Em conjunto essas glândulas produzem a saliva total na cavidade oral. A salivação é regulada por estímulos nervosos, principalmente sinais parassimpáticos. A estimulação simpática diminui a atividade glandular e o volume salivar. Outro fator que afeta a salivação é o suprimento de sangue nas glândulas salivares, que são sempre dependentes dos nutrientes adequados para a secreção. Os sinais parassimpáticos que induzem a salivação atuam também na vasodilatação da região glandular. É composta basicamente de água, moléculas orgânicas e eletrólitos. A porção aquosa 
da saliva vem da irrigação capilar local, passando da corrente sanguínea para as células secretoras por difusão (ZUARDI, 2012).

O óxido nítrico se apresenta na saliva basicamente em duas formas: sob a forma de gás produzido pelas células de defesa (como neutrófilos, macrófagos e plaquetas) contra bactérias e processos inflamatórios presentes na boca, e sob a forma líquida, convertida e estável de nitrito proveniente da redução de nitratos e da oxidação do próprio gás óxido nítrico. O nitrito presente na saliva pode vir de vias diferentes: da digestão de folhas, como alface, couve, espinafre, que produz grande quantidade de nitrato que é absorvido e da corrente sanguínea retorna à boca secretado pelas glândulas salivares e é rapidamente reduzido a nitrito pela ação da enzima nitrato redutase. Pode vir também da oxidação do gás óxido nítrico produzido na cavidade oral por células de defesa (TAKAHAMA, HIROTA \& TAKAYUKI, 2008). Tem sido demonstrado também que os nitritos podem vir da corrente sanguínea juntamente com o componente aquoso do sangue que chega às células secretoras das glândulas salivares, expressando assim, na saliva, as variações que este metabólito teria na corrente sanguínea (CLODFELTER et al, 2015).

A associação entre o NO sanguíneo ou plasmático e o salivar é o novo desafio dos pesquisadores dedicados ao estudo desde composto e sua ação no organismo humano. Os resultados de estudos dessa natureza são controversos e não têm identificado as variáveis envolvidas na controvérsia. Enquanto alguns estudos confirmam as variações de nitrito salivar com nitrito plasmático ou outras variáveis relevantes, como o exercício físico, outros estudos demonstram e afirmam a não associação entre nitrito salivar e plasmático, ou qualquer outra variável não relacionada diretamente à cavidade bucal. Algumas pesquisas relatam que o exercício físico não produz variações significativas nas concentrações de nitrito salivar, afirmando que não há associação entre essas duas variáveis (GONZÁLES et al, 2008), (ZAMBRANO et al, 2009), (CLODFELTER et al, 2015). Por outro lado, outros estudos relatam variações significativas na concentração de nitrito salivar a partir do exercício físico (PANOSSIAN et al, 1999), (DIAZ, et al, 2013). Nesses estudos são relatados aumento na concentração de
No salivar a partir do exercício, corroborando a hipótese de que o exercício, ao potencializar a produção de NO circulante, expressa esse mesmo potencial também na concentração de nitrito salivar. Há também relato de efeito inverso, isto é, o aumento da produção de NO circulante estimulada pelo exercício, acompanhado pela diminuição na concentração do nitrito salivar (MORAES, 2014), (AGRICOLA, 2016). Ao que tudo indica, há muito o que se descobrir sobre as interações entre o exercício físico e o óxido nítrico, as variações de intensidade e frequência do exercício, a produção de NO em condições adversas de saúde, os protocolos a serem testados. Enfim, a pesquisa nessa temática está apenas começando.

\section{REFERÊNCIAS}

AGRICOLA, N.P.A. Parâmetros hematológicos e concentração de óxido nítrico salivar em atletas de jiu jitsu: um estudo obsevacional. Goiânia / GO, tese (Doutorado), Universidade Federal de Goiás - Faculdade de Medicina (FM), 2016.

BARRETO, R. de L.; CORREIA, C.R.D. Óxido nítrico:propriedades e potenciais usos terapêuticos. Quim. Nova, Vol. 28, No. 6, 1046-1054, 2005.

BRANDÃO, A.F.; MARTINS-PINGE, M.C. Nitric oxide alteration in the cardiovascular function by exercise training. Semina: Ciências Biológicas e da Saúde, Londrina, v. 28, n. 1, p. 53-68, jan./jun. 2007.

BRUM, P.C.; SILVA, G.J.J.; MOREIRA, E.D.; IDA, F.; NEGRÃO, C.E.; KRIEGER, E.M. Exercise training increases baroreceptor gain sensitivity in normal and hypertensive rats. Hypertension, Dallas, v.36, n.6, p.1018- 1022, dec. 2000.

CERQUEIRA, N.F.; YOSHIDA, W.B. Óxido nítrico: revisão. Acta Cir Bras [serial online] 17 (6), Nov-Dez, 2002.

CLODFELTER, W.H.; BASU, S.; BOLDEN, C.; DOS SANTOS, P.C.; KING, S.B.; KIM-SHAPIRO, D.B. The relationship between plasma and salivary NOx. Nitric Oxide. 21;47: 85-90, Apr. 2015. http://dx.doi:10.1016/j.niox.2015.04.003.

CONGER, F.D. Endothelial regulation of vascular tone. Hosp Pract, 15:117-26. 1994.

DIAZ, M.M.; BOCANEGRA, O.L.; TEIXEIRA, R.R.; SOARES, S.S.; ESPINDOLA, F.S. Salivary nitric oxide and alpha-amylase as indexes of training intensity and load. Int J Sports Med. 34 (1): 8-13, Jan. 2013. http://dx.doi:10.1055/s-0032-1316318. 
DREXLER, H.; HORNIG, B. Endothelial dysfunction in human disease. J Mol Cell Cardiol. 31: 51-60, 1999.

DUSSE,L.M.S.; VIEIRA, L.M.; CARVALHO, M.G. Nitric oxide revision - Review Article. Jornal Brasileiro de patologia e medicina laboratorial. Rio de Janeiro, v.39, n 4, p. $343-$ 350, 2003.

DYAKOVA, E.Y.; KAPILEVICH, L.V.; SHYLKO, V.G.; POPOV, S.V.; ANFINOGENOVA, Y. Physical exercise associated with NO production: signaling pathways and significance in health and disease. Frontiers in Cell and Developmental Biology. Volume 3, Article 19, April, 2015; http://dx.doi: 10.3389/ fcell.2015.00019.

FLORA FILHO, R.; ZILBERSTEIN, B. Óxido nítrico: o simples mensageiro percorrendo a complexidade. Metabolismo, síntese e funções. Rev Ass Med Brasil, 46(3): 265-71, 2000.

FORJAZ, C.L.M.; SANTAELLA, D.F.; REZENDE, L.O.; BARRETTO, A.C.P.; NEGRÃO, C.E. A Duração do Exercício Determina a Magnitude e a Duração da Hipotensão Pós-Exercício. Arq Bras Cardiol, volume 70 ( $\left.n^{\circ} 2\right), 99-104,1998$.

FURCHGOTT, R.F.; ZAWADZKI, J.V. The obligatory role of endothelial cells in the relaxation of arterial smooth muscle by acetylcholine. Nature, 288: 373-6, 1980.

GONZÁLEZ, D.; MARQUINA, R.; RONDÓN, N.; RODRIGUEZ -MALAVER, A.J.; REYES, R. Effects of aerobic exercise on uric acid, total antioxidant activity, oxidative stress, and nitric oxide in human saliva. Res Sports Med. 16 (2): 128-37, 2008. http://dx.doi:10.1080/15438620802103700.

GRAHAM, D.A.; RUSH, J.W. Exercise training improves aortic endothelium-dependent vasorelaxation and determinants of nitric oxide bioavailability in spontaneously hypertensive rats. Journal of Applied Physiology, Bethesda, v.96, n.6, p.2088-2096, jun.2004.

HAMER, M. The anti-hypertensive effects of exercise: integrating acute and chronic mechanisms. Sports Medicine, Auckland, v.36, n.2, p.109-16. 2006.

KINGWELL, B.A. Nitric oxide-mediated metabolic regulation during exercise: effects of training in health and cardiovascular disease. FASEB J. 14: 1685-96, 2000.

KOSHLAND, J.R.D.E. The molecule of year. Science, 258:1861,1992.

KUBES, P.; GRANGER, D.N. Nitric oxide modulates microvascular permeability. American J Phys, 262: H611-H615, 1992.

MCARDLE, W. Fisiologia do Exercício: Energia nutrição e Desempenho humano. 5.ed. Rio de Janeiro: Guanabara Koogan. 2003.
MORAES, J.F.V.N. Associação entre nível de atividade física, nitrito salivar e fatores de risco cardiovascular em adolescentes portadores de variantes comuns dos genes FTO e ECA. Brasília / PUC, Tese de doutorado; 2014.

MURREL, G.A.C.; DOLAN, M.M.; JANG, D.; SZABO, C.; WARREN, R.F.; HANNAFIN, J.A. Nitric oxide: an important articular free radical. J Bone Joint Surg, 78-A:265-74,1996.

PAILLARD, T.; ROLLAND, Y.; BARRETO, P.S. Protective Effects of Physical Exercise in Alzheimer's Disease and Parkinson's Disease: A Narrative Review. J Clin Neurol, 11 (3): 212-219. 2015. http://dx.doi.org/10.3988/jcn.2015.11.3.212.

PANOSSIAN, A.G.; OGANESSIAN, A.S.; AMBARTSUMIAN, M.; GABRIELIAN, E.S.; WAGNER, H.; WIKMAN, G. Effects of heavy physical exercise and adaptogens on nitric oxide content in human saliva. Phytomedicine. 6 (1): 17-26, Mar, 1999.

PAULO, M. Efeito vasodilatador do doador de óxido nítrico [Ru(terpy)(bdq)NO $]^{+3}$ em veia cava e artéria basilar de ratos normotensos e hipertensos renais $2 \mathrm{R}-1 \mathrm{C}$. $\mathrm{Ri}$ beirão Preto: Tese de doutorado, USP, 159 p. 2011.

RAPAPORT, R.M.; MURAD, F. Agonist induced endothelium-dependent relaxation in rat thoracic aorta may be mediated throught cyclic GMP. Circ. Res. 52: 352-7, 1983.

SNYDER, S.H.; BREDT, D.S. Biological roles of nitric oxide. Scientific American, may: 28-35, 1992.

TAKAHAMA, U.; HIROTA, S.; TAKAYUKI, O. Detection of nitric oxide and its derivatives in human mixed saliva and acidified saliva. Methods Enzymol, 440: 381-96, 2008. http://dx.doi:10.1016/S0076-6879(07)00824-5.

WILMORE, J.H.; COSTIL, D.L. Physiology of Sport and Exercise. 2 ed. Champaign Ilinois, Human Kinetics. 2003.

WONG, G.K.T.; MARSDEN, P.A. Nitric oxide synthases: regulation in disease. Nefrol Dial Transplant, 11:215-20, 1996.

ZAGO, A.S.; ZANESCO, A. Óxido Nítrico, Doenças Cardiovasculares e Exercício Físico. Atualização Clínica. Arq Bras Cardiol, 87 (6) : e264-e270. 2006.

ZAMBRANO, J.C.; MARQUINA, R.; SULBARÁN, N.; RODRIGUEZ-MALAVER, A.J.; REYES, R.A. Aerobic exercise reduced oxidative stress in saliva of persons with Down syndrome. Res Sports Med. 17 (3): 195-203. 2009. http://dx. doi:10.1080/15438620903120843.

ZUARDI, L.R. Concentrações salivares, sanguíneas e plasmáticas de óxido nítrico em pacientes com doença periodontal antes e depois do tratamento periodontal não cirúrgico. Ribeirão Preto/USP, Dissertação de mestrado, 2012. 